\title{
Process, Structure, and Properties of Electrospun Carbon Nanotube-Reinforced Nanocomposite Yarns
}

\author{
Nasir M. Uddin, ${ }^{1}$ Frank Ko, ${ }^{2,3}$ Jie Xiong, ${ }^{4}$ Bakhtier Farouk, ${ }^{1}$ and Franco Capaldi ${ }^{1}$ \\ ${ }^{1}$ Department of Mechanical Engineering and Mechanics, Drexel University, Philadelphia, PA 19104, USA \\ ${ }^{2}$ Department of Materials Engineering, Drexel University, Philadelphia, PA 19104, USA \\ ${ }^{3}$ Department of Materials Engineering, University of British Columbia, 2355 East Mall, Vancouver, BC, Canada V6T $1 Z 4$ \\ ${ }^{4}$ College of Materials and Textiles, Zhejiang University of Sciences and Technology, West District, Xiasha Higher Education Zone, \\ Hangzhou 310018, China
}

Correspondence should be addressed to Bakhtier Farouk, bfarouk@coe.drexel.edu

Received 25 February 2009; Accepted 26 June 2009

Recommended by Jeffrey Glass

Carbon nanotubes (CNTs) are dispersed into polyacrylonitrile polymer solution and then assembled into continuous nanocomposite yarns through the drum-tape co-electrospinning process to facilitate the translation of CNT properties to higher order structures. We explore the dispersion of CNTs in a polymer matrix, the process of obtaining continuous yarn through electrospinning, and the surface morphology and mechanical properties of the nanocomposite yarn.

Copyright (C) 2009 Nasir M. Uddin et al. This is an open access article distributed under the Creative Commons Attribution License, which permits unrestricted use, distribution, and reproduction in any medium, provided the original work is properly cited.

\section{Introduction}

A new class of engineered nanomaterials with superior properties (modulus of about 1.0 TPa [1]) has emerged due to the discovery of carbon nanotubes (CNTs) by Iijima in 1991 [2]. The fabrication of carbon nanotube-CNTreinforced nanocomposite yarn is a promising addition to advanced composite materials. There are many promising applications for CNT-reinforced continuous polymer yarns. The creation of such continuous yarns would enable macroscopic nanotube devices and structures to be constructed using polymers as a matrix. While the yarn tends to have a micron scale diameter, it is composed of numerous nanoscale fibers naturally twisted together during processing. The process outlined in this paper could potentially lead to large scale production of nanofiber-reinforced planar and 3D fibrous structures such as woven, knitted, and braided fabrics, creating multiscale structures with optimized nano and macrostructures. It can also be extended to obtain CNT reinforced carbon fiber from the continuous polymer yarn. Donnet and Qin [3,4] showed that the properties of carbon fibers are mainly determined by the spinning procedure of precursor, rather than the precursor materials.
This study emphasizes the process, structure, and properties of obtaining continuous polymer yarn which could be used as precursor to obtain carbon fiber.

Electrospinning is a process by which ultrafine fibers with diameter on the nanometer length scale can be manufactured. Fibers are drawn using electrostatic forces from a polymer solution. Fiber diameter may be adjusted by varying the electric field strength and polymer solution concentration, whereas the duration of electrospinning controls the thickness of the deposited fiber mat [5]. Commonly, nanofibers are collected in the form of 2D fibrous nonwoven mats; linear fiber assemblies (yarns) can be produced. It has been shown by Ko et al. $[6,7]$ that nanofibrous yarns can be directly produced from the electrospinning process under well-controlled processing conditions for some polymers. Few research studies have focused on the creation and testing of CNT-reinforced polymer composite yarns. Fennessey and Farris [8] demonstrated the production of continuous yarns from electrospun PAN nanofibers using an electric twister. The process allowed the testing of the effects of twist on the properties of the yarn.

Dalton et al. [5] described the formation of electrospun poly(caprolactone) (PCL) fibers between two collection rings 


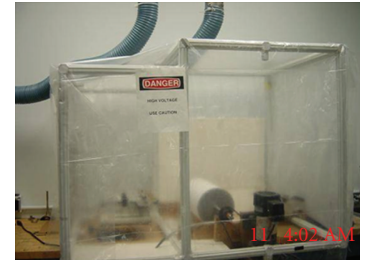

(a) Electrospinning station

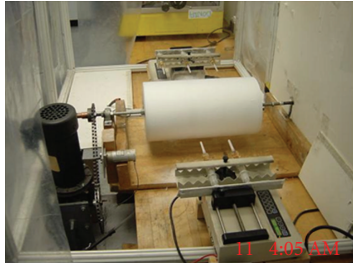

(b) electrospinning process setup
Figure 1: (a) Electrospinning station and (b) drum-tape electrospinning process setup.

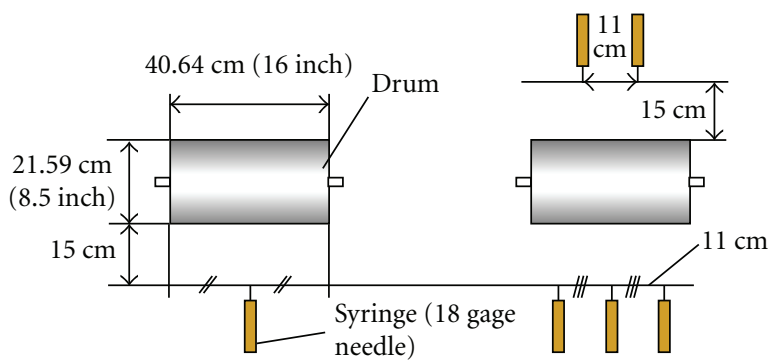

(a) Single syringe

(b) Five syringes

FIgURE 2: Dimensional configuration of the process.

and the conversion of these fibers into a multifilament yarn. They were able to obtain up to $50 \mathrm{~mm}$ long yarn. Smit et al. [9] described a technique for making continuous uniaxial fiber bundle yarns from electrospun fibers for very small scale production. Jiang et al. [10] showed that carbon nanotubes can be self-assembled into yarns of up to $30 \mathrm{~cm}$ in length simply by being drawn out from superaligned arrays of carbon nanotubes. A drum-tape electrospinning system has been developed in our laboratory for the creation of continuous yarn from electrospun nanofibers. The current study demonstrates the successful creation of continuous yarn and characterizes the level of dispersion of CNTs in the polymer matrix, the surface morphology, and the mechanical properties of nanocomposite yarns.

\section{Experiment}

1\% MWNT (Multiwalled Carbon Nanotube) is dispersed in Dimethyl-formamide (DMF) solvent by sonication, and 10\% PAN is added to the CNT/DMF by stirring. $1 \%$ of nonionic surfactant (polyethylene glycol, PEG) was added to decrease the agglomeration and improve the dispersion of the CNT as suggested by Vaisman et al. [11] and Pan et al. [12]. With the PEG treatment, the number of beads formed during electrospinning decreased, and a more uniform distribution of fiber diameter was observed.

The drum-tape electrospinning system has been developed in our laboratory as shown in Figure 1. Figure 1(a) shows the developed electrospinning station isolated from the surroundings and connected with an exhaust system.

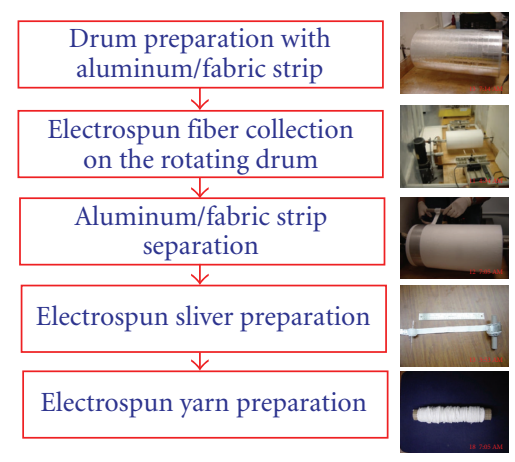

FIGURE 3: Process flow chart of obtaining continuous nanofiber yarn.

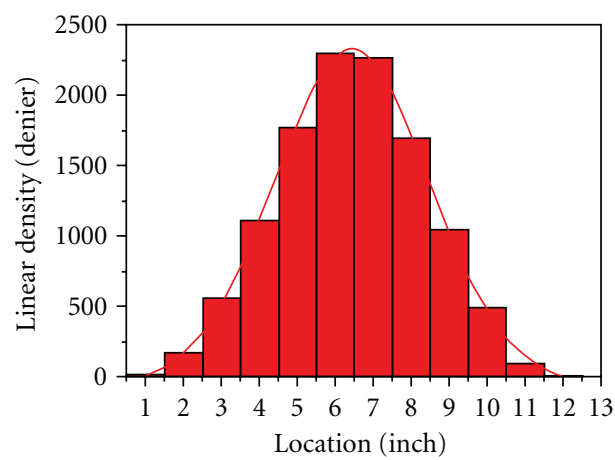

FIGURE 4: Linear density distribution of nanofiber yarn in case of single syringe.

Figure 2(b) describes the drum-tape electrospinning process setup showing the rotating drum and nozzle setup on both sides of the drum. In this system, a nonwoven mat of nanofibers is collected on aluminum foil/fabric tape wrapped on the rotating drum.

PAN fibers with diameters between $20 \mathrm{~nm}$ and $150 \mathrm{~nm}$ (investigated by the Scanning Electron Microscope) were electrospun by applying $20 \mathrm{kV}$ of electrical potential to the solution of PAN/MWCNT/DMF for about 12 hours with a flow rate of $0.5 \mathrm{~mL} / \mathrm{min}$. Electrospun fibers were collected onto the drum which was rotating at a linear surface speed between 3 and $5 \mathrm{~m} / \mathrm{s}$. Fibers were electrospun using both a single syringe as shown in Figure 2(a) and multiplesyringes as shown in Figure 2(b) on both sides of drum while maintaining a constant flow rate and drum speed.

The entire process of obtaining continuous yarns is illustrated by the process flow-chart shown in Figure 3. In this process, the drum is prepared by wrapping it with 1.0 inch aluminum foil strip in order to collect the electrospun fiber. After depositing enough nanofibers on the strip by electrospinning of the CNT-polymer solution, aluminum strip with the deposited aligned nanofiber is separated from the drum. Finally, the electrospun aligned nanofiber strip (sliver) is peeled off from the aluminum foil and twisted to make yarns (a linear assembly of fibers). 


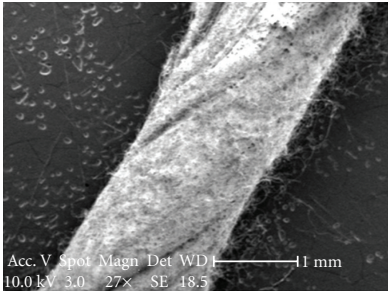

(a)

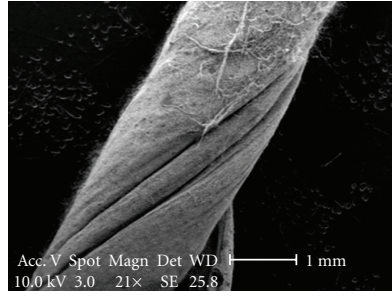

(b)
FIGURE 5: SEM images show the surface morphology of CNTreinforced PAN yarn of (a) poor dispersion and (b) improved dispersion.

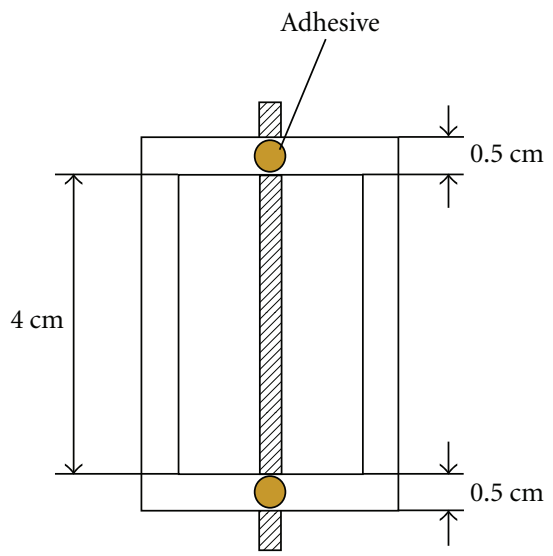

FIGURE 6: The specimen of nanofiber yarn for tensile test.

\section{Results and Discussion}

CNTs are dispersed into polyacrylonitrile (PAN) polymer solution using dimethylformamide as a solvent and then assembled into a continuous nanocomposite yarn through the drum-tape co-electrospinning process. The linear density distribution of nanofibers along the drum was examined. Figure 4 demonstrates that, near the center of the drum, the fiber deposition was higher when spun with a single syringe as shown in Figure 2(a) than that with the multiple syringes. A uniform distribution was obtained with fibers spun using multiple syringes as shown in Figure 2(b).

SEM (Scanning Electronic Microscope) was used to investigate the structural morphology of CNT-reinforced PAN nanofiber yarns formed with and without a surfactant added to the polymer solution. Figure 5(a) has poor CNT dispersion of CNT reinforced PAN yarn spun without surfactant, and we can see that much of it has aggregated on the surface and lends no reinforcement to the matrix. Figure 5(b) was spun from PAN with surfactant and has improved dispersion characteristics. Both samples contain the same filler concentration $(1 \mathrm{wt} \%)$. These fibers were tested in tension to investigate the mechanical response using the Kawabata KES-G1 tensile tester where we used an extension rate of $0.2 \mathrm{~mm} / \mathrm{s}$ and gage length as $40 \mathrm{~mm}$. The specimen of nanofiber yarn was prepared as shown in Figure 6. A paper frame with proper dimension was used for

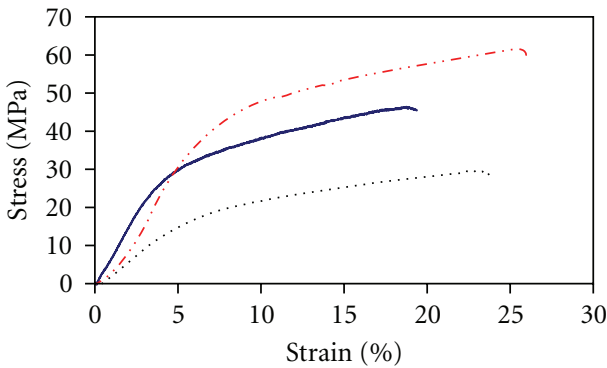

- PAN with 1\% MWCNT (poor dispersion)

-.. - PAN with 1\% MWCNT (improved dispersion)

- Pure PAN

FIGURE 7: Stress-strain relationship for CNT-reinforced composite yarn.

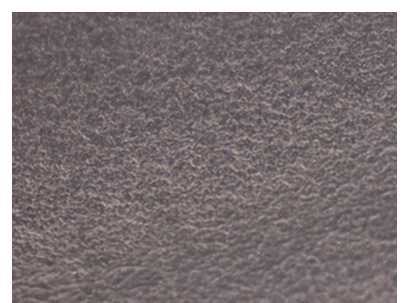

(a) PAN/CNT/DMF (poor dispersion)

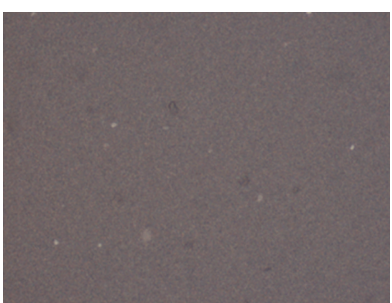

(b) $\quad$ PAN/CNT/DMF/PEG (improved dispersion
FIGURE 8: Optical microscope pictures (10x) of PAN/CNT dispersion.

mounting the nanofiber yarn sample between the jaws of the tester.

The stress-strain relationship for MWCNT-reinforced composite yarn is shown in Figure 7. The pure PAN nanofibers tensile strength is about $46 \mathrm{MPa}$ which is quite reasonable as compared with the measurements of Wang et al. [13]. It can be observed that the strength of CNTreinforced PAN nanofiber yarn is reduced by about $40 \%$ in the case of poor CNT dispersion into polymer. This guided us to improve the CNT dispersion with the addition of a surfactant. The yarn tensile strength increases by about 35\% due to MWCNT reinforcement in the PAN polymer with surfactant-aided CNT dispersion. The mechanical properties of the well-dispersed sample are improved relative to those of the poorly dispersed sample. Both toughness and the yield strength were increased significantly by improving dispersion. From this, we can see that dispersion must be controlled to optimize the reinforcement value for a given volume fraction of nanofiller.

The dispersion quality of CNT-polymer solution was determined by the optical microscope in the liquid state by collecting CNT-polymer solution on the glass-slide. Figure 8(a) shows the image of CNT-dispersed PAN polymer solution without added surfactant. The surfactant-aided CNT-dispersed PAN polymer solution image is shown in Figure 8(b). It was observed that the addition of surfactant decreased the CNT aggregates providing the smooth surface and ultimately improved dispersion. 


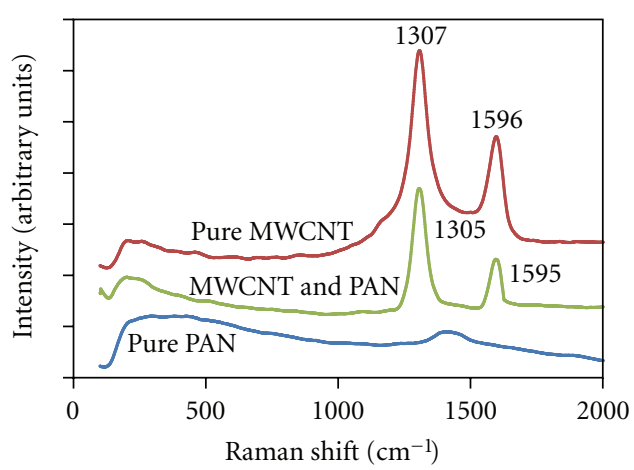

(a) Reinforcement of CNT in PAN nanofiber

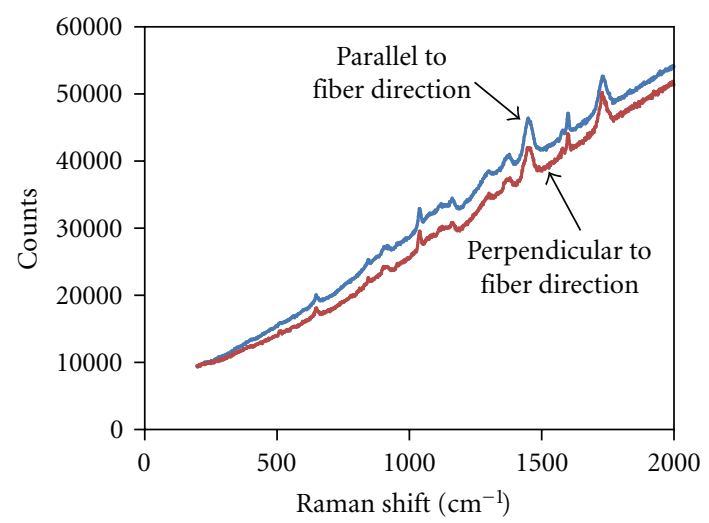

(b) Alignment of CNT in PAN nanofiber

FIGURE 9: Raman spectra for CNT-reinforced PAN yarn describe (a) the presence of CNT in nanofiber and (b) the alignment of CNT along the nanofiber's axial direction.

The presence and alignment of CNT in nanofiber yarns (Figure 9) were confirmed by using the polarized Raman Spectroscopy (Renishaw RM1000). Raman spectra were collected and analyzed for MWCNT, MWCNT-PAN nanofiber, and PAN nanofiber laid on a clean glass. Figure 9(a) demonstrates that the Raman band for MWCNT exists in the CNT-reinforced PAN nanofiber indicating the reinforcement of MWCNT in the PAN nanofiber. To illustrate the CNT alignment in nanofiber, the aligned nanofiber tows (the formed untwisted nanofibers in close proximity to one another), obtained from the sliver, Figure 3, were laid flat on a cleaned glass slide and then fixed on a rotating stage of Raman Spectrometer. This rotating stage was mounted on the microscope stage to allow rotation of the fibers relative to the incident light. The sample was first aligned parallel to the incident light, and images were obtained at $0^{\circ}$ and $90^{\circ}$. The CNT-alignment analysis was carried out using a $514.5 \mathrm{~nm}$ (Ar ion laser) excitation wavelength operating at 10\% power.

Figure 9(b) illustrates that the Raman spectra (VV configuration) from the same location on the sample are taken at two different angles $(\theta)$ relative to the incident light. When the light was polarized along the length of the nanotube, the intensities at all modes are at maximum $(\theta=$ $0^{\circ}$ ) and decrease when deviate from parallel and eventually become minimum at $\theta=90^{\circ}$. The decrease in intensity of the Raman signal as the relative angle between the polarized light and the tube axis increases suggested a high degree of MWCNT alignment in the nanofiber. It is hypothesized that the alignment of the CNTs in nanofiber occurs through three mechanisms: flow-confinement-and charge-induced alignment during the electrospinning process. This study concludes that in order to exploit the properties of CNTs in the nanocomposite microstructure, it is essential to better understand the process, structure, and properties of the composite yarn [7-10, 14-18].

\section{Conclusion}

This study has successfully demonstrated that CNTs can be incorporated in electrospun nanofibers. Nanofibers with aligned CNTs can be used to obtain continuous composite yarn. The drum-tape co-electrospinning process provides an important pathway to translate the properties of the nanoscale CNT to the macroscopic structures. It is clear that CNT load transfer into polymer matrix is dependent upon the degree of dispersion which needs to be explored further.

\section{References}

[1] J.-M. Bonard, J.-P. Salvetat, T. Stöckli, L. Forró, and A. Châtelain, "Field emission from carbon nanotubes: perspectives for applications and clues to the emission mechanism," Applied Physics A, vol. 69, no. 3, pp. 245-254, 1999.

[2] S. Iijima, "Helical microtubules of graphitic carbon," Nature, vol. 354, no. 6348, pp. 56-58, 1991.

[3] J.-B. Donnet and R.-Y. Qin, "Study of carbon fiber surfaces by scanning tunneling microscopy-part II: pan-based high strength carbon fibers," Carbon, vol. 31, no. 1, pp. 7-12, 1993.

[4] J.-B. Donnet and R.-Y. Qin, "Study of carbon fiber surfaces by scanning tunnelling microscopy-part I: carbon fibers from different precursors and after various heat treatment temperatures," Carbon, vol. 30, no. 5, pp. 787-796, 1992.

[5] P. D. Dalton, D. Klee, and M. Möller, "Electrospinning with dual collection rings," Polymer, vol. 46, no. 3, pp. 611-614, 2005.

[6] F. K. Ko, S. Khan, A. Ali, et al., "Structure and properties of carbon nanotube reinforced nanocomposites," in Proceedings of the 43rd Structures, Structural Dynamics and Materials Conference, vol. 3, pp. 1779-1787, Denver, Colo, USA, April 2002.

[7] F. Ko, Y. Gogotsi, A. Ali, et al., "Electrospinning of continuous carbon nanotube-filled nanofiber yarns," Advanced Materials, vol. 15, no. 14, pp. 1161-1165, 2003.

[8] S. F. Fennessey and R. J. Farris, "Fabrication of aligned and molecularly oriented electrospun polyacrylonitrile nanofibers and the mechanical behavior of their twisted yarns," Polymer, vol. 45, no. 12, pp. 4217-4225, 2004.

[9] E. Smit, U. Búttner, and R. D. Sanderson, "Continuous yarns from electrospun fibers," Polymer, vol. 46, no. 8, pp. 24192423, 2005.

[10] K. Jiang, Q. Li, and S. Fan, "Spinning continuous carbon nanotube yarns," Nature, vol. 419, no. 6909, p. 801, 2002.

[11] L. Vaisman, H. D. Wagner, and G. Marom, "The role of surfactants in dispersion of carbon nanotubes," Advances in Colloid and Interface Science, vol. 128-130, pp. 37-46, 2006. 
[12] C. Pan, L.-Q. Ge, and Z.-Z. Gu, "Fabrication of multi-walled carbon nanotube reinforced polyelectrolyte hollow nanofibers by electrospinning," Composites Science and Technology, vol. 67, no. 15-16, pp. 3271-3277, 2007.

[13] X. Wang, K. Zhang, M. Zhu, B. S. Hsiao, and B. Chu, "Enhanced mechanical performance of self-bundled electrospun fiber yarns via post-treatments," Macromolecular Rapid Communications, vol. 29, no. 10, pp. 826-831, 2008.

[14] X.-L. Xie, Y.-W. Mai, and X.-P. Zhou, "Dispersion and alignment of carbon nanotubes in polymer matrix: a review," Materials Science and Engineering R, vol. 49, no. 4, pp. 89-112, 2005.

[15] Y. Dror, W. Salalha, R. L. Khalfin, Y. Cohen, A. L. Yarin, and E. Zussman, "Carbon nanotubes embedded in oriented polymer nanofibers by electrospinning," Langmuir, vol. 19, no. 17, pp. 7012-7020, 2003.

[16] O. Breuer and U. Sundararaj, "Big returns from small fibers: a review of polymer/carbon nanotube composites," Polymer Composites, vol. 25, no. 6, pp. 630-645, 2004.

[17] L. Jin, C. Bower, and O. Zhou, "Alignment of carbon nanotubes in a polymer matrix by mechanical stretching," Applied Physics Letters, vol. 73, no. 9, pp. 1197-1199, 1998.

[18] A. Theron, E. Zussman, and A. L. Yarin, "Electrostatic fieldassisted alignment of electrospun nanofibres," Nanotechnology, vol. 12, no. 3, pp. 384-390, 2001. 

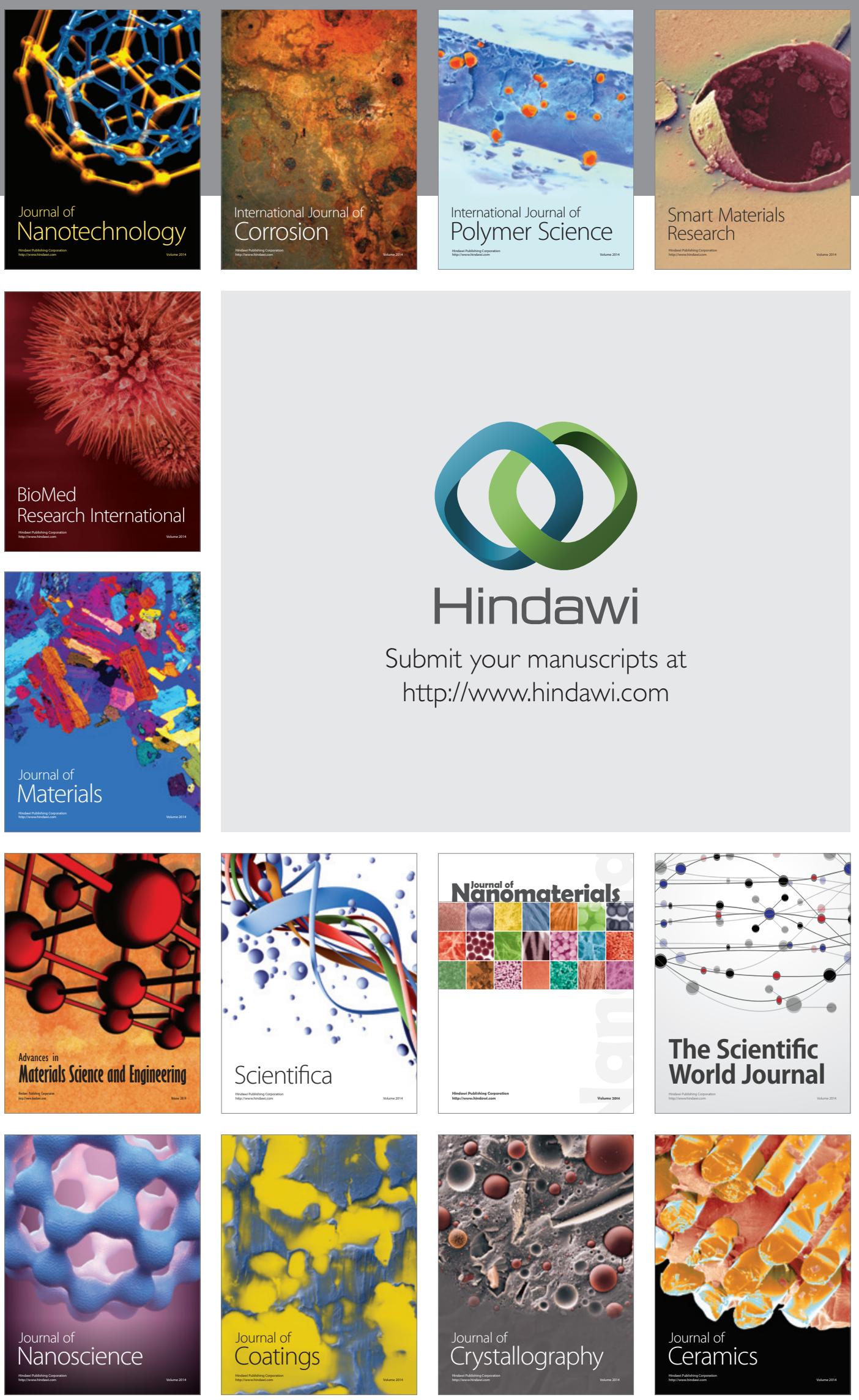

The Scientific World Journal

Submit your manuscripts at

http://www.hindawi.com

\section{World Journal}

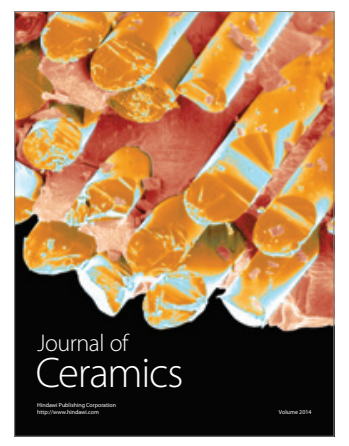

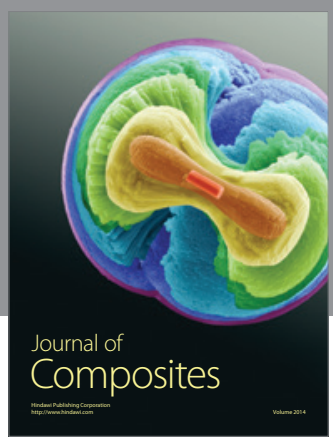
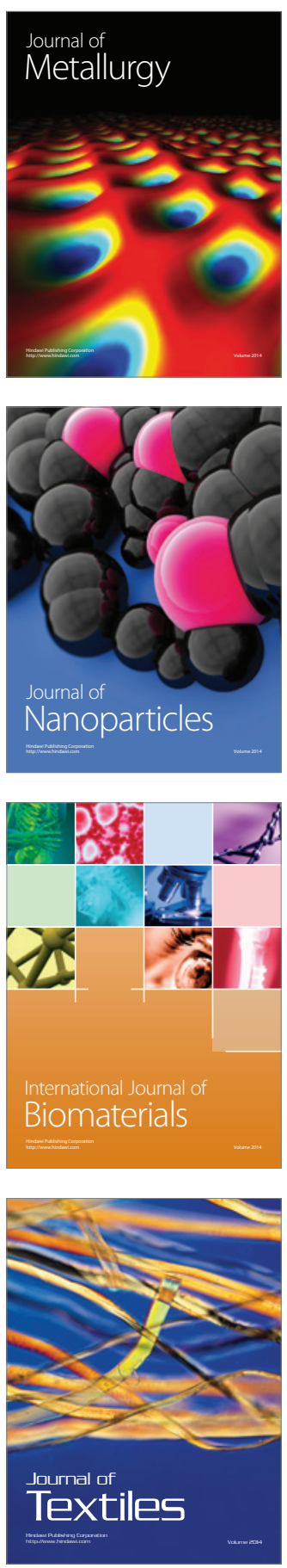